\title{
Numerous HPV warts in a young boy with Netherton syndrome: A case report.
}

\author{
Armaghan Ghareaghaji Zare ${ }^{1}$, Afsane Radmehr ${ }^{1}$, Leila Sadat Hatamnezhad ${ }^{1}$, Siamak Sandoghchian \\ Shotorbani $^{*}$
}

${ }^{1}$ Department Of Dermatology, Sina Hospital, Tabriz University Of Medical Sciences, Tabriz, Iran

${ }^{2}$ Department of Immunology, faculty of medicine, Tabriz University Of Medical Sciences, Tabriz, Iran

\begin{abstract}
Netherton's syndrome (NS) is a rare disorder characterized by dermatological signs, such as ichthyosis, and a complex immunological dysfunction. Human papillomavirus (HPV) infection is associated with carcinomas on non-mucosal sites in immunodeficient individuals. We report a case of a 15-year-old boy with itchy, red skin, and fragile scalp and eyebrow hairs. HPV warts were diagnosed through the clinical findings and laboratory analyses. Leukocytosis with mild eosinophilia and normal hemoglobin was confirmed using blood test analyses. After diagnosis, we presented some treatment options.
\end{abstract}

Keywords: HPV, Netherton syndrome, Warts

Accepted on November 25, 2017

\section{Introduction}

Comel Netherton syndrome is a severe, autosomal recessive form of ichthyoids associated with mutations in the SPINK5 gene that encodes the serine protease inhibitor lympho-epithelial Kazaltype-related inhibitor (LEKTI) [1]. Such mutations result in a dysfunctional protein with a reduced capacity to inhibit serine proteases expressed in the skin. KLK5, KLK7, and KLK14 serve as potential endogenous targets of LEKTI [2]. These enzymes are involved in various aspects of epidermal remodeling, including desquamation, PAR-2 activation, and degradation of lipid hydrolases, suggesting a potential mechanism to develop atopic manifestations of Netherton syndrome [3]. Patients with Netherton syndrome tend to have a hair shaft defect (trichorrhexis invaginata, also known as "bamboo hair"), congenital ichthyosis, erythroderma atopy, food allergies, and asthma [4]. In severe cases, these atopic manifestations persist throughout the individual's life, resulting in high post-natal mortality rates [5]. In contrast, less severe patients develop the milder ichthyosis linearis circumflexa. Patients with Netherton syndrome exhibit an increased susceptibility to viral skin infections, specifically Herpes simplex virus (HSV) and human papillomavirus (HPV) [6]. Patients with netherton syndrome show high levels of IgE, which is normal at birth, increases with increasing age, and then decreases over time. Warts are commonly found in the genital area of patients with Netherton syndrome [7]. Some types of HPV have the potential to progress to carcinoma. It is important to note that patients with EV-associated HPV types have been found in biopsy and skin cancers. Genital warts are soft growths appearing on the genitals [8], which are a sexually transmitted infection (STI) caused by certain strains of HPV. HPV can cause squamous cell carcinoma (SCC) [9]. Cutaneous SCC is the most common tumor affecting post-transplant patients. Certain molecular phenotypes are able to distinguish post-transplant skin SCCs from immuno-competent patients such as high levels of p53 and TGFB, as well as low levels of phosphorylated mTOR and P70S6K [10]. These skin growths can cause pain, discomfort, and itching. HPV strains are especially dangerous for women because some types of HPV can cause cancer of the cervix and vulva [11]. Treatments based on monoclonal and polyclonal antibodies containing corticosteroids were found to be most effective. In addition, IVIG $(0.4 \mathrm{~g} / \mathrm{kg})$ and anti TNF $\alpha$ antibodies (Infliximab) were shown to have strong activity.

\section{Case Report}

We report here a case of a 15-year-old boy who was born with itchy, red skin, sparse, and fragile scalp and eye brow hair. After birth, his skin became more ichthyosiform and erythroderma with marked lichenification (Figure 1).

There was no evidence or history of typical polycyclic scaly patch with double aged scales. Numerous HPV warts were detected on the patient's face, causing a cobblestrone pattern. These lesions specially were observed on scalp. Pathology findings showed that the involuting lesions may have chronic inflammatory infiltrates in dermis and epidermis with degenerative epithelial changes (Figure 2). Immunoglobulin levels and lymphocyte subsection profiling showed down-regulation of Natural Killer T-cells (NKT) and CD8+ lymphocytes, in combination with increased levels of serum immunoglobulins. We performed no functional immunological tests, such as granulocyte function, natural killer-cell and peripheral blood mononuclear cell proliferation assays. Some HPV warts were also on the hands and forearm, but not on the groins and genital skin. The patient had the personal history of food allergy since childhood. For more study of this case, a sample of rough and lusterless hair (bamboo hairs) was taken and evaluated under a polarized light microscope (Figure 3).

Blood test analyses showed that leukocytosis was characterized with increased eosinophilia (15200/mic); however, Hb, ESR, and FBS levels were in the normal range. Results from the blood test showed that the IgE level was high than $2000 \mathrm{IU} / \mathrm{ml}$ (Table 1). These characteristic findings led to the diagnosis of Comel Netherton syndrome that had not been diagnosed before in this patient.

\section{Discussion}

This is one of the very few case reports on HPV warts in 
Citation: Zare G, Radmehr A, Hatamnezhad LS, et al. Numerous HPV warts in a young boy with Netherton syndrome: A case report. Biol Med Case Rep. 2018;2(1):1-3.
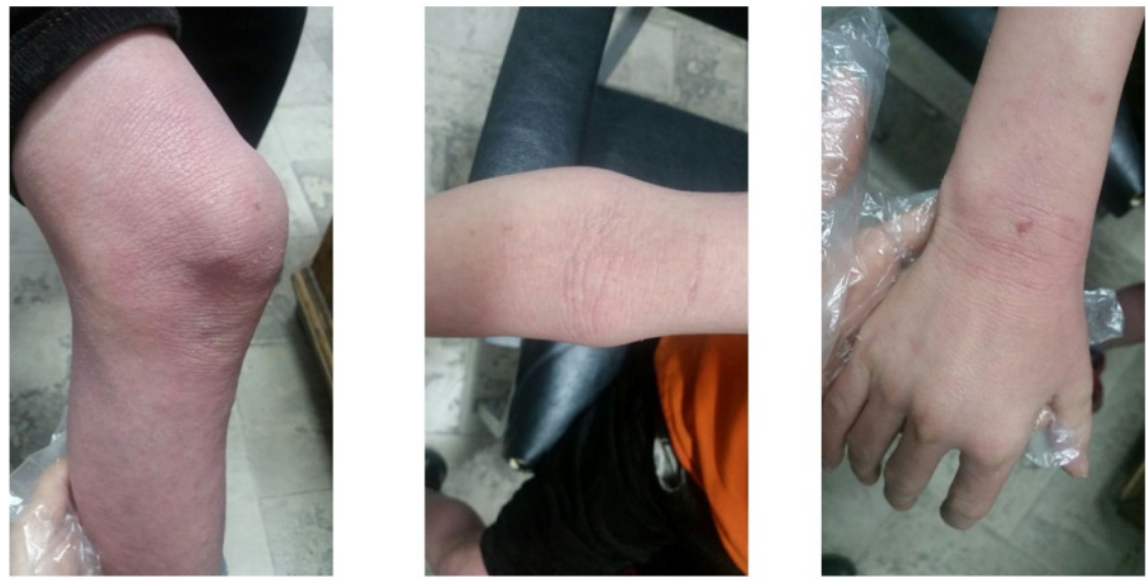

Figure 1. Ichthyosiform erythroderma and lichenification.

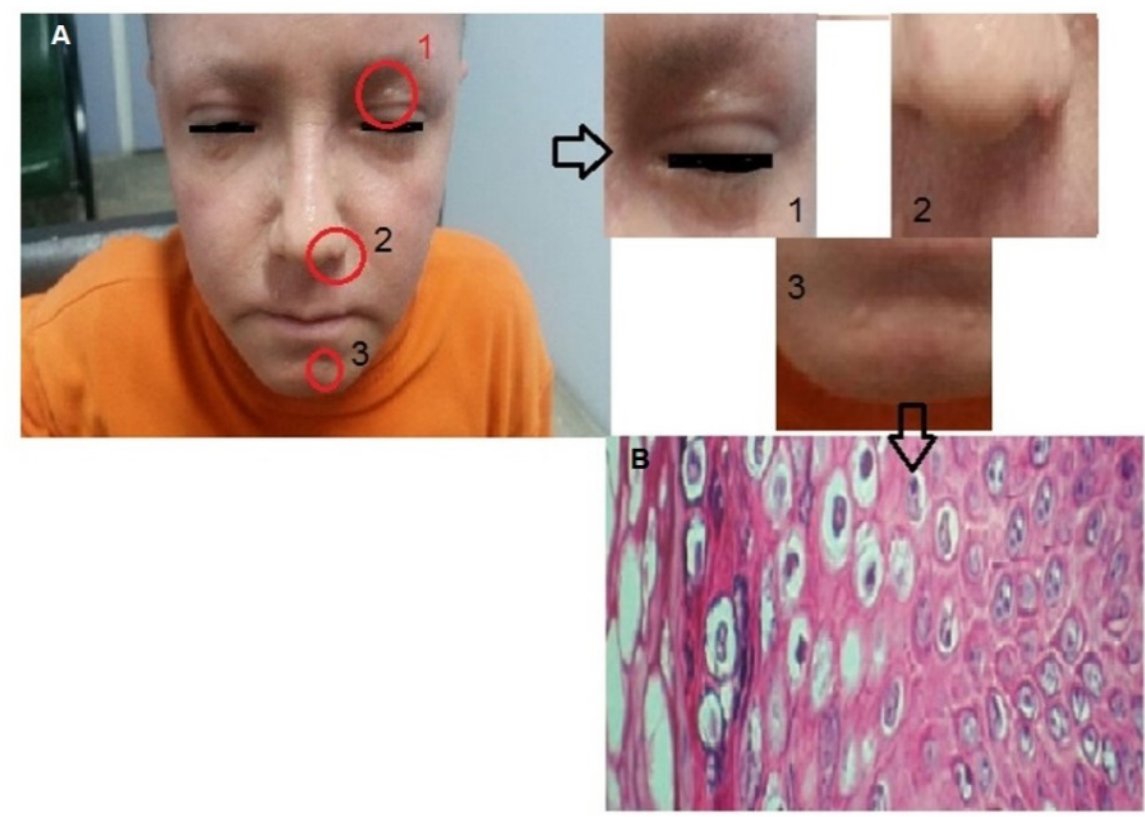

Figure 2. (A) Numerous HPV warts, (B) Pathology findings showed that the involuting lesions may have chronic inflammatory infiltrates in dermis and epidermis with degenerative epithelial changes.
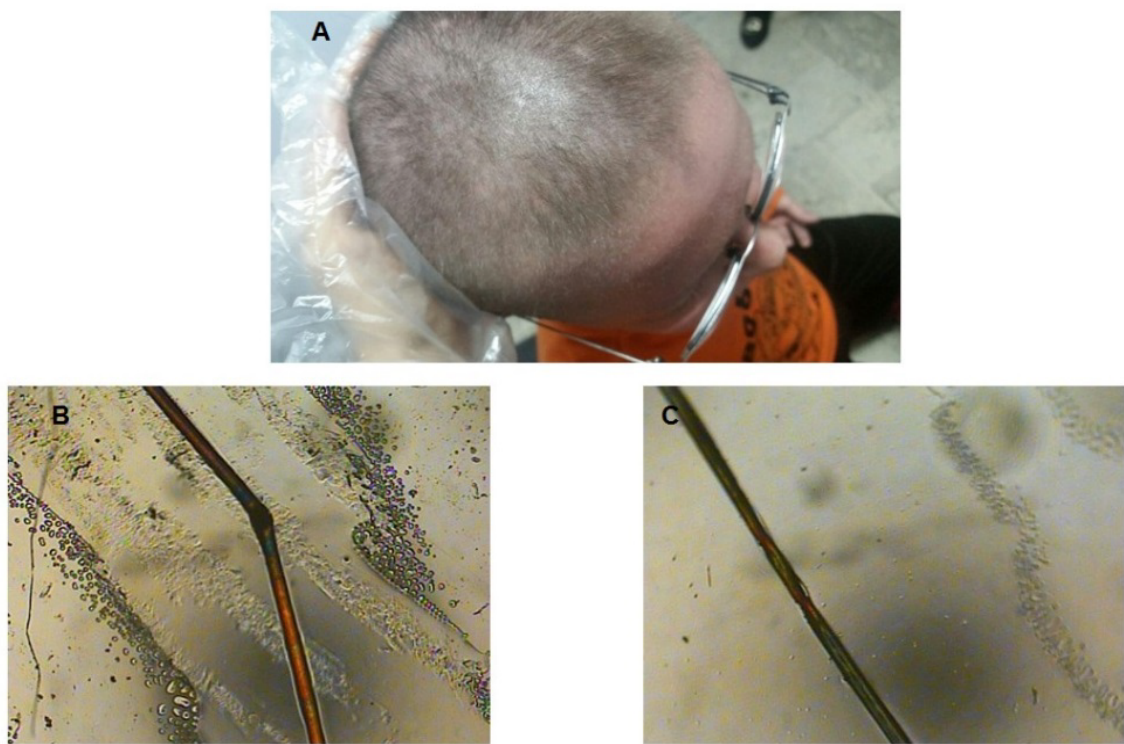

Figure 3. (A) Rough and lusterless hair; (B) Bamboo hairs, (C) Pili torti). 
Table 1. Laboratory analysis.

\begin{tabular}{|c|c|c|}
\hline Hematology & Result/Unit & References \\
\hline WBC & 15200/micro L & $4000-11000$ \\
\hline RBC & $4.69 \mathrm{MIL} / \mathrm{Mic}$ & $4.5-5.9$ \\
\hline $\mathrm{Hb}$ & $14 \mathrm{~g} / \mathrm{dl}$ & $13.5-18$ \\
\hline HCT & $43.3 \%$ & $40-54$ \\
\hline M.C.V & $92.3 f l$ & $80-96$ \\
\hline M.C.H & 29.9 pic & $27-33$ \\
\hline $\mathrm{MCHC}$ & $32.3 \%$ & $31-35$ \\
\hline Platelets & $476000 / \mathrm{Micl}$ & $150-450 \times 10^{6}$ \\
\hline RSR $1 \mathrm{~h}$ & $3 \mathrm{~mm} / \mathrm{hr}$ & $3-10$ \\
\hline Biochemestry & -- & -- \\
\hline Glu & 91 & -- \\
\hline $\mathrm{HbA1C}$ & 5.1 & -- \\
\hline Blood Urea & 8.4 & -- \\
\hline Urea & 18 & -- \\
\hline Creatinine & .6 & -- \\
\hline Cholesterol Total & 181 & -- \\
\hline Triglisiride & 72 & -- \\
\hline LDL & 61 & -- \\
\hline HDL & 106 & -- \\
\hline SGOT & 19 & -- \\
\hline SGPT & 19 & -- \\
\hline
\end{tabular}

patients suffering from Netherton syndrome. Most studies discussed HPV warts and their relation with tumors, but a few ones investigated HPV warts and their relationship with Netherton syndrome [12]. In a study in 2001, Weber et al. showed the relationship among HPV warts, TGF-B and NKT cells in SCC [13]. Ella et al. demonstrated Netherton Syndrome with multiple non-melanoma skin cancers with expression of SPINK-5 genes [14]. Nicole et al. exhibited HPV Carcinomas in Immunocompromised Patients [15]. They showed that vulvar cancer accounts for approximately $5 \%$ of all gynecological cancers, showing the involvement of HPV in this cancer [15]. The anogenital warts in men are associated with a 5- to 6-fold increased risk of penile SCC. One study reported the histological comparison of HPVrelated SCC of the scrotum from non-HPV-related SCC of the scrotum, which their test was positive for p16 and Ki67, while the non-HPV-related SCC of the scrotum was consistently negative for p16 and had variable Ki67 expression [16]. Ella et al. reported the case report of a 39-year-old patient with NS who developed multiple skin malignancies in Netherton syndrome [14].

\section{Conclusion}

The present case report discussed HPV warts and Neuterthon syndrome with high expression of IgE and high WBC count. Rough and lusterless hairs were also observed in this report. Ichthyosiform and erythroderma, as important symptoms of this disease, were also found in this case report. For treatment, we recommended IVIG $(0.4 \mathrm{~g} / \mathrm{kg})$ and anti TNF $\alpha$ antibodies (Infliximab), which show strong effectiveness. It would be beneficial to investigate the effectiveness of currently-available treatments in this same patient population.

\section{References}

1. Saif GB, Al-Khenaizan S. Netherton syndrome: Successful use of topical tacrolimus and pimecrolimus in four siblings. Int J Dermatol. 2007;46:290-294.
2. Hartschuh W, Hausser I, Petzoldt D. Erfolgreiche Retinoidtherapie des Netherton-Syndroms. Hautarzt. 1989;40:430-433.

3. Braun RP, Ramelet AA. Failure of cyclosporine in Netherton's syndrome. Dermatology. 1997;195:75.

4. Oji V, Beljan G, Beier K, et al. Topical pimecrolimus: A novel therapeutic option for Netherton syndrome. Br J Dermatol. 2005; 153:1067-1068.

5. Renner ED, Hartl D, Rylaarsdam S, et al. Comel-Netherton syndrome defined as primary immunodeficiency. J Allergy Clin Immunol. 2009;124:536-543.

6. Maatouk I, Moutran R, Tomb R. Narrowband ultraviolet B phototherapy associated with improvement in Netherton syndrome. Clin Exp Dermatol. 2012;37:364-366.

7. Kaminska EC, Ortel B, Sharma V, et al. Narrowband UVB phototherapy as a novel treatment for Netherton syndrome. Photodermatol Photoimmunol Photomed. 2012;28:162-164.

8. Capezzera $\mathrm{R}$, Venturini $\mathrm{M}$, Bianchi $\mathrm{D}$, et al. UVA1 phototherapy of Netherton syndrome. Acta Derm Venereol. 2004;84:69-70.

9. Bingol B, Tasdemir S, Gunenc Z, et al. Prenatal diagnosis of Comel-Netherton syndrome with PGD, case report and review article. J Assist Reprod Genet. 2011;28:615-620.

10. Krasagakis K, Ioannidou DJ, Stephanidou M, et al. Early development of multiple epithelial neoplasms in Netherton syndrome. Dermatology. 2003;207:182-184.

11. Natsuga $K$, Akiyama $M$, Shimizu H. Malignant skin tumours in patients with inherited ichthyosis. Br J Dermatol. 2011;165:263-268.

12. Saghari S, Woolery-Lloyd H, Nouri K. Squamous cell carcinoma in a patient with Netherton's syndrome. Int J Dermatol. 2002;41:415-416.

13. Weber F, Fuchs PG, Pfister HJ, et al. Human papillomavirus infection in Netherton's syndrome. $\mathrm{Br} \mathrm{J}$ Dermatol. 2001;144:1044-1049.

14. Van der Voort EAM, Errol P. Netherton syndrome with multiple non-melanoma skin cancers. Acta Derm Venereol. 2013;93:727-728.

15. Reusser NM. HPV carcinomas in immuno-compromised patients. J. Clin. Med. 2015;4:260-281.

16. Ormerod AD. Topical tacrolimus and pimecrolimus and the risk of cancer: How much cause for concern? Br J Dermatol. 2005;153:701-705.

\section{*Correspondence to:}

Siamak Sandoghchian Shotorbani

Faculty of Medicine

Department of Immunology

Tabriz University of Medical Sciences

Tabriz

Iran

Tel/Fax: +41-4791331

E-mail: siamak1331@gmail.com 